\title{
Elevated Vascular Endothelial Growth Factor Level in Association with Mean Platelet Volume Are Emerging Risk Factors for Vascular Complications in T2DM Patients
}

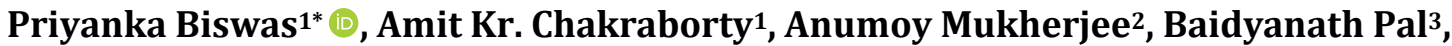 \\ Debasish Maji ${ }^{4}$, Madhusnata De ${ }^{1}$

\begin{abstract}
${ }^{1}$ Department of Genetics, Vivekananda Institute of Medical Sciences, Ramakrishna Mission Seva Pratishthan, Kolkata, India
${ }^{2}$ Department of Pathology, Murshidabad Medical College and Hospital, Berhampore, India

${ }^{3}$ Biological Anthropological Unit, Indian Statistical Institute, Kolkata, India

${ }^{4}$ Department of Endocrinology, Vivekananda Institute of Medical Sciences, Ramakrishna Mission SevaPratishthan, Kolkata, India

Email: *priyan_bsws@yahoo.com, amitdod@gmail.com, anumoy.rgkmch@gmail.com, baidya@isical.ac.in,

d.maji50@gmail.com, madhusnata_de@yahoo.com
\end{abstract}

\begin{abstract}
How to cite this paper: Biswas, P., Chakraborty, A.K., Mukherjee, A., Pal, B., Maji, D. and De, M. (2019) Elevated Vascular Endothelial Growth Factor Level in Association with Mean Platelet Volume Are Emerging Risk Factors for Vascular Complications in T2DM Patients. Health, 11, 1542-1553.
\end{abstract}

https://doi.org/10.4236/health.2019.1111116

Received: August 30, 2019

Accepted: November 25, 2019

Published: November 28, 2019

Copyright $\odot 2019$ by author(s) and Scientific Research Publishing Inc. This work is licensed under the Creative Commons Attribution International License (CC BY 4.0).

http://creativecommons.org/licenses/by/4.0/

\section{(c) (i) Open Access}

\begin{abstract}
Increasing evidence in both experimental and clinical studies suggests oxidative stress (OS) plays a major role in the pathogenesis of type 2 diabetes mellitus and its complications. In a physiological condition, appropriate levels of ROS, generated either in restricted amounts or transient fashion, are required to promote physiological angiogenesis and homeostatic maintenance of healthy vasculature. Uncontrolled continuous ROS production will ultimately contribute to pathology and cause tissue damage. One of the most important proangiogenic factors is vascular endothelial growth factor (VEGF) which plays a key role in diabetic endothelial dysfunction, which ultimately leads to pathogenesis of vascular complications. As VEGF is released by activated platelets, hence platelet activation could be the source of VEGF in plasma samples. Increased platelet activity is emphasized to play a role in the development of vascular complications in T2DM patients and platelet volume, a marker of the platelet function and activation, is measured as mean platelet volume (MPV). Therefore, we aimed to investigate the association between plasma VEGF levels and MPV levels that may lead to vascular complications. A case-control study of one hundred patients with T2DM $(\mathrm{n}=100)$ and thirty control subjects $(n=30)$ were screened from different areas of West Bengal. All procedures were done with the informed consent of participants. Elevated VEGF level was found in T2DM patients $(526.8 \pm 34.3 \mathrm{pg} / \mathrm{ml})$ compared to healthy controls $(317.9 \pm 12.9 \mathrm{pg} / \mathrm{ml})$ which was statistically signifi-
\end{abstract}


cant. T2DM patients had higher MPV $(12.5 \pm 0.2 \mathrm{fl})$ compared to controls $(11.4 \pm 0.2 \mathrm{fl})$. Thus the present study showed a significant association between VEGF level in plasma and MPV indicating the severity of vascular complications. Hence, in conclusion, it suggested that VEGF levels along with MPV are a reliable biomarker for evaluating the development and progression of vascular complications.

\section{Keywords}

Oxidative Stress, Vascular Endothelial Growth Factor, Mean Platelet Volume, Vascular Complications

\section{Introduction}

Type 2 diabetes mellitus (T2DM), the most frequent subtype of diabetes, comprises a complex heterogeneous group of metabolic diseases characterized by high levels of blood glucose (hyperglycaemia) and impaired insulin action and/or insulin secretion. Anticipating an epidemic like increase in the number of diabetic patients, India is being referred as the "diabetic capital of the world" [1]. About 31.0\% populations of West Bengal are suffering from T2DM [2]. The incidence of T2DM varies substantially from one geographical region to the other as a result of environmental and lifestyle risk factors [3].

One of the factors associated with diabetic complications is the process of non-enzymatic glycation of proteins, lipids and nucleic acids, with subsequent formation of advanced glycation end products (AGEs) [4]. One of the main consequences of this adverse action is the formation of oxidative stress (OS). Formation of glycation end products leads to OS and subsequent DNA damage via the oxidation of DNA bases and sugar-phosphate binding sites. OS represents an imbalance between the production and manifestation of reactive oxygen species (ROS) especially free radicals and a biological system's ability to detoxify the reactive intermediates or to repair the resulting damage.

In a physiological condition, appropriate levels of ROS, generated either in restricted amounts or transient fashion, are required to promote physiological angiogenesis and homeostatic maintenance of healthy vasculature. In normal circumstances i.e. during physiological conditions, the formation of new blood vessels occurs during wound healing, organ regeneration and in the female reproductive system during ovulation, menstruation and the formation of the placenta. Uncontrolled continuous ROS production will ultimately contribute to pathology and cause tissue damage. During physiological or repair processes, such as wound healing, the inflammation process is transient; most pathological conditions involve a continuous recruitment of inflammatory cells, which, in turn, serve as substantial source of ROS [5]. OS-dependent angiogenesis is an important contributor to the progression of cancers and chronic diseases. Hyperglycemia-induced diacylglycerol, a lipid molecule, activates protein kinase 
$\mathrm{C}$ in the vascular tissues leading to the formation of OS and the latter in turn promotes VEGF signaling, resulting in diabetic microvascular complications [6]. Vascular endothelial growth factor (VEGF) is the most potent proangiogenic growth factor that increases vascular permeability in vivo and activates endothelial cells in vitro [7].

Other factors responsible for induction of VEGF are failure of $\beta$-cell function and the deterioration of systemic insulin sensitivity. Many investigations suggested that the reason behind $\beta$-cell failure in T2DM is the decreased $\beta$-cell mass and the dysfunction of islets. The pancreatic islets are highly vascular tissues and their dysfunctions of vascular structure lead to deterioration of insulin secretion [8]. Expression of vascular endothelial growth factor (VEGF) in $\beta$-cells is associated with the formation of vascular structure. Clinically, T2DM is involved with failure of various organs, especially the blood vessels and can lead to many complications over time. These complications include retinopathy, neuropathy, nephropathy, impaired wound healing and accelerated atherosclerosis which are implicated in a large number of cellular and subcellular changes in vessels. VEGF may play a key role in diabetic endothelial dysfunction, which ultimately leads to pathogenesis of vascular complications. Many studies reported that plasma VEGF levels were higher in diabetic patients compared to healthy control individuals and a correlation of plasma VEGF levels with late diabetic complications had also been documented [9]-[11]. However, there are few investigations illustrating the detailed mechanism of the association between VEGF and late diabetic complications i.e. vascular complications.

As VEGF is released by activated platelets, hence platelet activation during blood collection could be an artificial source of VEGF in plasma samples. A correlation could occur between VEGF levels in plasma samples and blood platelet activation at the time of blood sampling. This may explain conflicting results in studies of VEGF plasma levels in DM and other conditions with endothelial dysfunction [12]. Increased platelet activity is emphasized to play a role in the development of vascular complications in T2DM patients and platelet volume, a marker of the platelet function and activation, is measured as mean platelet volume (MPV). T2DM patients have an increased risk for the development of late diabetic complication i.e. microvascular and macrovascular complications and platelets play an important role in alteration of platelet morphology and function [13].

For better understanding, T2DM patients with or without complications and healthy controls were selected and their VEGF plasma levels were assessed in this study. For further confirmation, MPV was measured in the diabetics compared to the healthy controls to determine if platelets were activated in diabetes and in its associated vascular complications for assurance of VEGF release. Furthermore, no such study was found in the literature which sought to investigate the relationship between plasma VEGF levels in the context of type 2 diabetes mellitus and its complications in population of West Bengal. Therefore, we aimed to investigate the association between plasma VEGF levels and MPV 
levels that may lead to vascular complications.

\section{Methods}

\subsection{Study Setting and Subjects}

A total of one hundred patients with T2DM and thirty healthy control subjects were recruited from different areas of West Bengal. The participants were age and sex-matched. The patients were confirmed of having T2DM by a level of glycosylated hemoglobin ( $\mathrm{HbAlc}$ ) of $\geq 6.5 \%$, impaired fasting glucose test ( $>126$ $\mathrm{mg} / \mathrm{dl})$ and oral glucose tolerance test $(>200 \mathrm{mg} / \mathrm{dl})$. The controls had a self reported history of having normal glucose metabolism. Patients meeting any of the above criteria can be categorized as T2DM. The control category selected healthy participants without any type of diabetes, as well as without hypertension, hyperlipidemia and other metabolic syndromes. We had excluded the patients who had fever, acute and chronic infections, malignancy, acute and chronic nephritis, cirrhosis and congestive heart failure. All the patients were under stable conditions during assessment. Detailed personal histories were collected from the participants with the help of questionnaire.

This study was approved by the institutional ethical committee on $10^{\text {th }}$ May, 2016. All procedures were done with the informed consent of participants. The methods in this study were performed in accordance with the relevant guidelines and regulations.

\subsection{Collection of Peripheral Blood}

Peripheral blood samples were collected by venipuncture both from T2DM patients and control subjects. Blood samples were collected in EDTA vacutainer and stored at $4^{\circ} \mathrm{C}$ for experimental work.

\subsection{Measurement of Plasma VEGF}

$3 \mathrm{ml}$ of peripheral blood samples were collected from the patients and healthy volunteers to determine the plasma VEGF level. Plasma was separated from these blood samples by centrifugation at $2500 \mathrm{rpm}$ for 20 minutes.

The plasma VEGF concentration was determined using Human VEGF (Vascular Endothelial Growth Factor) enzyme-linked immunosorbent assay kit (Fine Test, China) in Bioradi Mark ${ }^{\mathrm{TM}}$ Microplate Reader. Intra-assay precision was < $8 \%$ and Inter-assay precision was $<10 \%$. The detection range of the assay was 0 $2000 \mathrm{pg} / \mathrm{ml}$.

\subsection{Protocol of Haematological Study}

The Platelet count, Platelet Distribution Width (PDW) and Mean Platelet Volume (MPV) were estimated by Sysmex K-2000 automated cell counter.

\subsection{Statistical Analysis}

The statistical evaluation was performed using statistical software Statistical 
Package for Social Sciences (SPSS). All data were expressed as mean \pm SE (Standard Error). The statistical significance was determined based on a probability level of less than 0.05. For comparison of the VEGF levels between T2DM patients and healthy controls, independent t-test was performed. The correlations between the levels of VEGF and other variables were calculated using Pearson's correlation coefficient.

\section{Results}

Socioeconomic status, particularly income and educational qualification are important factors in higher prevalence of T2DM. Diabetes may be up to two times more prevalent in low income populations compared to wealthy populations [14] [15] [16].

Income level, which is a major reflection of socioeconomic status (SES), has a close association with the adverse health effects including the incidence of T2DM. SES is a complex concept that describes the position, which an individual occupies in the structure of society. SES has been recognized as an important determinant of a population's health. SES is closely linked to a wide range of health problems, including communicable and non-communicable diseases, with different strengths and directions of association in different populations [17]. Many studies have seldom reported the discrepancy of T2DM incidence between different SES groups in developing or recently developed economies, especially in Asia. In addition to their disproportionate prevalence of diabetes, individuals with low SES tend to have poorer glycemic control, more diabetes complications and higher mortality rate. Lack of access to health care has been considered as an important risk factor for the consequences of diabetes among the socioeconomically deprived apart from personal vulnerability and other predisposing factors [18]. According to Table 1, most of the T2DM patients had pattern of higher prevalence towards low monthly household income of Rs. 5001 - Rs. 10,000 p.m. which leads to the discrepancy in the quality of diabetes care. Income disparity appears to predispose people with T2DM towards inequality in diabetes care, which includes delayed identification of cases and inadequate follow-up, even in a nation with a comprehensive universal health insurance program. Thus this study indicates that comprehensive and universal health coverage can lead to improvement of access which is merely a start toward eliminating inequality in diabetes care.

Many investigations reported that there is a strong relationship between SES

Table 1. Distribution of monthly income of studied cases.

\begin{tabular}{|c|c|c|c|c|c|c|c|c|c|}
\hline \multirow{2}{*}{$\begin{array}{c}\text { Type of } \\
\text { Individuals }\end{array}$} & \multirow{2}{*}{$\begin{array}{l}\text { Total No. of } \\
\text { Individuals }\end{array}$} & \multicolumn{2}{|c|}{ <Rs. 5000 P.M. } & \multicolumn{2}{|c|}{$\begin{array}{l}\text { Rs. } 5001 \text { - Rs. } \\
\text { 10,000 P.M. }\end{array}$} & \multicolumn{2}{|c|}{$\begin{array}{c}\text { Rs. 10,001 - Rs. } \\
\text { 15,000 P.M. }\end{array}$} & \multicolumn{2}{|c|}{ >Rs. 15,000 P.M. } \\
\hline & & No. & $\%$ & No. & $\%$ & No. & $\%$ & No. & $\%$ \\
\hline Healthy Control & 30 & 1 & 3.3 & 5 & 16.7 & 15 & 50 & 9 & 30 \\
\hline T2DM & 100 & 16 & 16 & 56 & 56 & 23 & 23 & 5 & 5 \\
\hline
\end{tabular}


and health outcomes in patients with T2DM. SES refers to the social position of an individual relative to other members of a society which cannot be measured directly. Instead, different proxies for SES are used and often involve educational level, income or occupation. These three indicators are strongly associated and complementary, but are not interchangeable. The major advantage of using educational level as a proxy for SES is its simplicity and universality [19]. It is suggested that health outcomes (which show the effects of care on the health status of patients) are influenced by different distal factors involving individual (eg, cultural background) or community characteristics, as well as by more proximal factors such as health behaviors, access to health care and processes of care for a patient. Most studies related to the relationship between SES and processes of diabetes care reported worse processes of care among patients of lower SES. It is well accepted that processes of care influence diabetes outcomes and many processes are considered indicators of quality of diabetes care. Thus, achieving optimal processes of care is postulated to be a key element in the care of patients with diabetes [20]. A direct comparison to these findings is, however, rather difficult, as almost every study selected different sets of processes and outcomes of care. Our results nevertheless remain consistent with the literature as most of the cases had school level education (primary or high school) according to Table 2. Thus higher percentage of T2DM cases prevailed at low educational level. This depicts that a better education may contribute to increased quality of life through better understanding of the nature of the disease, better coping with the disease and eventually better diabetes care overall.

Table 3 demonstrated that Mean platelet volume (MPV), Platelet distribution width (PDW) and total platelet count were elevated in T2DM compared to healthy controls and platelet distribution width was statistically significant.

Comparisons between Control and T2DM cases in terms of Glucose concentration following Impaired Fasting Glucose and Oral Glucose Tolerance Test have been graphically presented in Figure 1(a) and Figure 1(b) respectively [ ${ }^{*}$ Statistically significant at $p \leq 0.05$ (Independent $t$-test)].

Comparison between Control and T2DM cases in terms of HbAIC (\%) has been presented graphically in Figure $2\left[{ }^{*}\right.$ Statistically significant at $p \leq 0.05$ (Independent $t$-test)].

VEGF levels in plasma were compared between healthy controls and T2DM. Elevated VEGF level was found in T2DM patients compared to healthy controls and it was found statistically significant (Table 4).

Table 2. Distribution of education of studied cases.

\begin{tabular}{cccccccccccc}
\hline \multirow{2}{*}{ Types } & $\begin{array}{c}\text { Total no. of } \\
\text { Individuals }\end{array}$ & \multicolumn{2}{c}{ Illiterate } & \multicolumn{2}{c}{$\begin{array}{c}\text { Primary Education } \\
(<\text { Class VIII) }\end{array}$} & $\begin{array}{c}\text { High School } \\
\text { (Class VIII - Class XII) }\end{array}$ & \multicolumn{2}{c}{$\begin{array}{c}\text { College } \\
\text { (Graduation })\end{array}$} & \multicolumn{2}{c}{$\begin{array}{c}\text { Higher Education } \\
(\geq \text { Postgraduation })\end{array}$} \\
\cline { 3 - 12 } & & No. & $\%$ & No. & $\%$ & No. & $\%$ & No. & $\%$ & No. & $\%$ \\
\hline $\begin{array}{c}\text { Healthy Control } \\
\text { T2DM }\end{array}$ & 30 & 0 & 0 & 3 & 10 & 5 & 16.7 & 13 & 43.3 & 9 & 30 \\
\hline
\end{tabular}


Table 3. Haematological status of studied cases.

\begin{tabular}{ccccc}
\hline $\begin{array}{c}\text { Types of } \\
\text { Individuals }\end{array}$ & No. Of Individuals & $\begin{array}{c}\text { Platelet count }\left(\times 10^{3} / \mu \mathrm{l}\right) \\
(\text { Mean } \pm \text { S.E. })\end{array}$ & $\begin{array}{c}\text { PDW (\%) } \\
(\text { Mean } \pm \text { S.E) }\end{array}$ & $\begin{array}{c}\text { MPV (fl) } \\
(\text { Mean } \pm \text { S.E.) }\end{array}$ \\
\hline Controls & 30 & $191.8 \pm 11.2$ & $12 . \pm 0.2$ & $11.4 \pm 0.2$ \\
T2DM & 100 & $197.4 \pm 5.7$ & $\begin{array}{c}16.5 \pm 0.1 \\
p=0.000\end{array}$ & $12.5 \pm 0.2$ \\
\hline
\end{tabular}

Table 4. VEGF level in plasma in studied cases.

\begin{tabular}{ccc}
\hline Group & VEGF Level in plasma $(\mathrm{pg} / \mathrm{ml})($ mean $\pm \mathrm{SE})$ & $p$-value \\
\hline Healthy Controls & $317.9 \pm 12.9$ & - \\
T2DM & $526.8 \pm 34.3$ & $0.000^{* *}$ \\
\hline
\end{tabular}

*Statistically significant at $p \leq 0.001$ (Independent $t$-test).

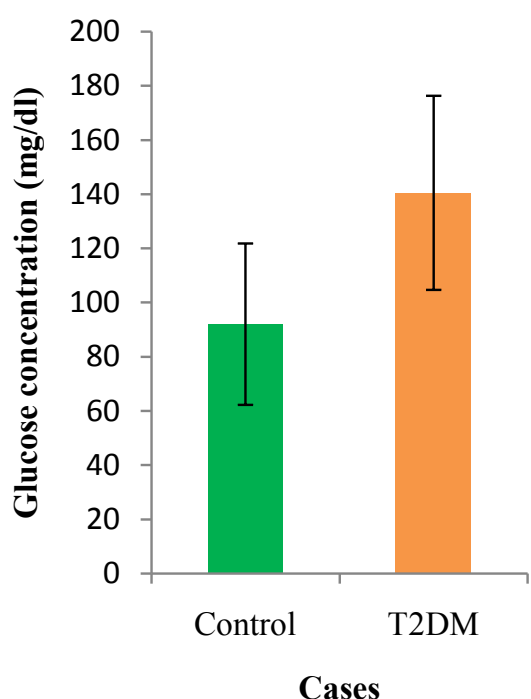

(a)

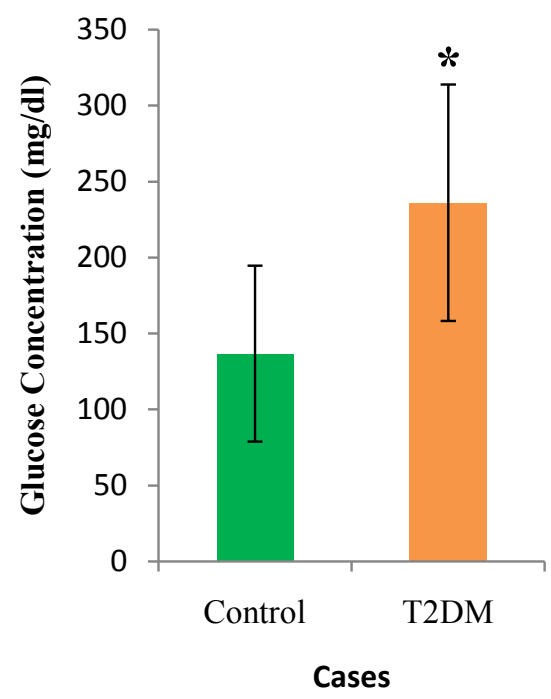

(b)

Figure 1. Comparison between Control and T2DM cases in terms of Glucose concentration following impaired fasting glucose and oral glucose tolerance test.

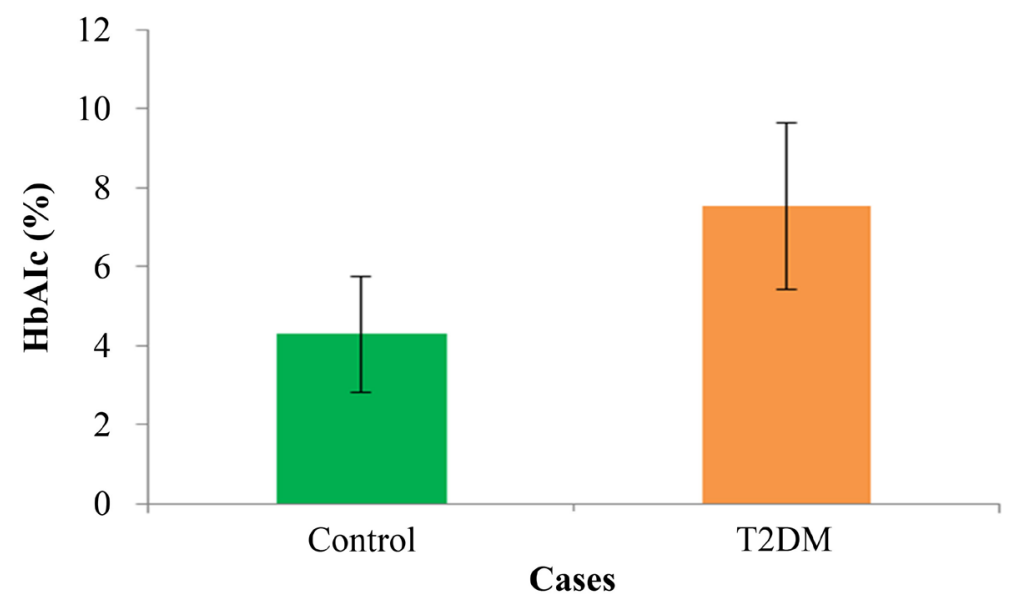

Figure 2. Comparison between control and T2DM cases in terms of HbAIC (\%). 
Pearson correlation coefficients were calculated to analyze the associations between plasma VEGF level and mean platelet volume (MPV), platelet distribution width (PDW) and total platelet count. Table 5 demonstrated that VEGF levels were correlated positively with MPV $(r=0.196, p<0.05)$ and PDW $(r=$ $0.266, p<0.01$ ) in the T2DM patients and controls indicating that VEGF is associated with increased mean platelet volume and platelet distribution width.

\section{Discussion}

The number of diabetes cases worldwide has increased to approximately 366 million according to the estimation by the International Diabetes Federation, of which T2DM constitutes approximately $90 \%$ to $95 \%$. Up to $80 \%$ of mortality with T2DM is directly associated with vascular complications (microvascular complications or macrovascular complications) [21]. Angiogenesis i.e. the formation and differentiation of blood vessels, is an essentially biological process existing in embryogenesis and in the development of late diabetic complications [22]. Vascular endothelial growth factor (VEGF) is a major mediator of angiogenesis and plays a key role in the pathogenesis of late diabetic complications. VEGF is an endothelial cell specific mitogen, has been implicated as a major contributor to the development of diabetic complications and plays an important role in the pathogenesis of endothelial dysfunction. VEGF expression in endothelial vascular leads to increase in permeability of blood vein that induces increased angiogenesis activity. VEGF is also associated with tumor progression that may lead to cancer [23] [24]. The activated platelets are the main sources of VEGF in blood. It has been well documented that platelets are a rich source of

Table 5. Relationships between VEGF level and its putative determinants.

\begin{tabular}{|c|c|c|c|c|c|}
\hline \multicolumn{6}{|c|}{ Correlations } \\
\hline & & VEGF & PDW & MPV & Platelet \\
\hline \multirow{3}{*}{ VEGF } & Pearson Correlation & 1 & $0.266^{\star *}$ & $0.196^{*}$ & -0.141 \\
\hline & Sig. (2-tailed) & & 0.002 & 0.026 & 0.109 \\
\hline & $\mathrm{N}$ & 130 & 130 & 130 & 130 \\
\hline \multirow{3}{*}{ PDW } & Pearson Correlation & $0.266^{\star *}$ & 1 & $0.343^{\star *}$ & -0.016 \\
\hline & Sig. (2-tailed) & 0.002 & & 0.000 & 0.857 \\
\hline & $\mathrm{N}$ & 130 & 130 & 130 & 130 \\
\hline \multirow{3}{*}{ MPV } & Pearson Correlation & $0.196^{*}$ & $0.343^{\star *}$ & 1 & $-0.196^{*}$ \\
\hline & Sig. (2-tailed) & 0.026 & 0.000 & & 0.025 \\
\hline & $\mathrm{N}$ & 130 & 130 & 130 & 130 \\
\hline \multirow{3}{*}{ Platelet } & Pearson Correlation & -0.141 & -0.016 & $-0.196^{*}$ & 1 \\
\hline & Sig. (2-tailed) & 0.109 & 0.857 & 0.025 & \\
\hline & $\mathrm{N}$ & 130 & 130 & 130 & 130 \\
\hline
\end{tabular}

${ }^{\star *}$ Correlation is significant at the 0.01 level (2-tailed); ${ }^{\star}$ Correlation is significant at the 0.05 level (2-tailed). 
VEGF which is released upon their activation and more the degree of platelets are activated, more the VEGF is released. Mean Platelet Volume (MPV) and platelet counts are indicators of platelet activation and thrombotic potentials and independent risk factors for late diabetic complications. MPV is an indicator of the average size and activity of platelets, with a higher MPV value indicating a larger average platelet size. Larger platelets are younger, more reactive and aggregable. Hence, they contain denser granules, secrete more serotonin and $\beta$-thromboglobulin and produce more thromboxane A2 than smaller platelets. All these can produce a pro-coagulant effect and cause thrombotic vascular complications [25]. This leads to a relationship between the platelet function especially MPV and diabetic vascular complications thus indicating changes in MPV that reflects the state of thrombogenesis. There might be small bleeds due to the rupture of atherothrombotic plaques leading to increased platelet recruitment, hyper reactivity and bone marrow stimulation. High MPV is emerging as a new risk factor for the vascular complications of DM of which atherothrombosis plays a major role. Thus, DM has been considered as a "prothrombotic state" with increased platelet reactivity [13].

It is interesting that the correlations of VEGF with MPV, platelet count and PDW are found in our cohort study, suggesting an interaction that may exist among vascular complications and T2DM patients. Thus the present study showed a significant association between VEGF level in plasma and MPV, PDW indicating the severity of vascular complications. Elevated VEGF level was found in T2DM patients $(526.8 \pm 34.3 \mathrm{pg} / \mathrm{ml})$ compared to healthy controls $(317.9 \pm$ $12.9 \mathrm{pg} / \mathrm{ml}$ ) which was statistically significant. T2DM patients had higher MPV $(12.5 \pm 0.2 \mathrm{fl})$ compared to controls $(11.4 \pm 0.2 \mathrm{fl})$ which suggested a role for the increased platelet activity in the pathogenesis of vascular complications. Our findings were similar to the studies done by Shinichiro et al. and Ates et al. where MPV was positively correlating with the degree of late diabetic complications in their cases [25] [26]. This indicates that elevated VEGF and MPV are risk factors for late diabetic complications and they have a strong relationship. Therefore, the positive correlation between VEGF levels and MPV leads to severity of vascular complications in T2DM. To best of our knowledge, this is the first cohort study to investigate the association between VEGF, MPV and incident diabetes risk leading to vascular complications.

The pathophysiological mechanism for increased VEGF expression involving in the development and progression of vascular complications is not yet fully understood. However, several possible reasons may account for their association. First, over-expression of VEGF induced by persistent hyperglycemia can lead to increasing vascular endothelium permeability leading to angiogenesis. Second, increasing evidence indicates inflammation leading to OS which plays a key role in the development of late complications and VEGF is a strong inducer of inflammation [27]. Several studies demonstrated that VEGF plays an essential and causative role in retinal inflammation [27] [28]. Therefore, over-expression of VEGF exacerbates inflammatory reaction which might be responsible for the 
progression of late diabetic complications.

\section{Conclusion}

In conclusion, we observed that elevated VEGF levels in plasma are a risk factor for the presence and severity of vascular complications in diabetic patients which is supported by elevated MPV and a strong correlation exists between them. This suggests that VEGF levels along with MPV are a reliable biomarker for evaluating the development and progression of vascular complications. Thus, we propose that VEGF level in association with MPV can be used as a simple and cost-effective tool to monitor the progression and control of T2DM and its vascular complications. The outcome of our study can help the clinicians to modify their treatment policy according to their patient needs. However, the increased VEGF level and MPV as the cause or the end result of vascular complications need to be further explored. The recruitment of participants conducted in the present study was limited, therefore, an extensive study with increased sample size is recommended.

\section{Acknowledgements}

I would like to acknowledge our Secretary, Ramakrishna Mission Seva Pratishthan, colleagues of our department and all the patients who participated in this study.

\section{Author Statements}

Ethical approval: This study was approved by the institutional ethical committee on $10^{\text {th }}$ May, 2016. Reg No. ECR/62/Inst/WB/2013 issued under Rule 122DD of the Drugs \& Cosmetics Rules 1945.

\section{Funding}

Department of Science \& Technology, West Bengal.

\section{Conflicts of Interest}

None declared.

\section{References}

[1] Joshi, A.R., Pranita, A. and Phadke, A.V. (2012) Diabesity: Curse of Development. International Journal of Medicine, 12, 77-78.

[2] Shashank, R.J., Banshi, S., Muruga, V., et al. (2012) Prevalence of Diagnosed and Undiagnosed Diabetes and Hypertension in India-Results from the Screening India's Twin Epidemic (SITE) Study. Diabetes Technology \& Therapeutics, 12, 8-15. https://doi.org/10.1089/dia.2011.0243

[3] Zimmet, P., Alberti, K.G. and Shaw, J. (2001) Global and Societal Implications of the Diabetes Epidemic. Nature, 414, 782-787. https://doi.org/10.1038/414782a

[4] Brownlee, M. (1994) Glycation and Diabetic Complications. Diabetes, 43, 836-841. https://doi.org/10.2337/diab.43.6.836 
[5] Schreml, S., Szeimies, R.M., Prantl, L., Karrer, S., Landthaler, M. and Babilas, P. (2010) Oxygen in Acute and Chronic Wound Healing. British Journal of Dermatology, 163, 257-268. https://doi.org/10.1111/j.1365-2133.2010.09804.x

[6] Pathak, D., Gupta, A., Kamble, B., Kuppusamy, G. and Suresh, B. (2012) Oral Targeting of Protein Kinase C Receptor: Promising Route for Diabetic Retinopathy? Current Drug Delivery, 9, 405-413. https://doi.org/10.2174/156720112801323080

[7] Asselbergs, F.W., Boer, R.A., Diercks, G.F. et al. (2004) Vascular Endothelial Growth Factor: The Link between Cardiovascular Risk Factors and Microalbuminuria? International Journal of Cardiology, 93, 211-215.

https://doi.org/10.1016/j.ijcard.2003.04.001

[8] Iwashita, N., Uchida, T., Choi, J.B., Azuma, K., Ogihara, T., Ferrara, N., Gerber, H., Kawamori, R., Inoue, M. and Watada, H. (2007) Impaired Insulin Secretion in Vivo But Enhanced Insulin Secretion from Isolated Islets in Pancreatic Beta Cell-Specific Vascular Endothelial Growth Factor-A Knock-Out Mice. Diabetologia, 50, 380-389. https://doi.org/10.1007/s00125-006-0512-0

[9] Costa, P.Z. and Soares, R. (2013) Neovascularization in Diabetes and Its Complications. Unraveling the Angiogenic Paradox. Life Sciences, 92, 1037-1045. https://doi.org/10.1016/j.lfs.2013.04.001

[10] Hovind, P., Tarnow, L., Oestergaard, P.B., et al. (2000) Elevated Vascular Endothelial Growth Factor in Type 1 Diabetic Patients with Diabetic Nephropathy. Kidney International Supplements, 75, S56-S61. https://doi.org/10.1046/j.1523-1755.57.s75.4.x

[11] Ma, Y., Zhang, Y., Zhao, T., et al. (2012) Vascular Endothelial Growth Factor in Plasma and Vitreous Fluid of Patients with Proliferative Diabetic Retinopathy Patients after Intravitreal Injection of Bevacizumab. American Journal of Ophthalmology, 153, 307-313. https://doi.org/10.1016/j.ajo.2011.08.006

[12] Schlingemann, R.O., Van Noorden, C.J., Diekman, M.J., et al. (2013) VEGF Levels in Plasma in Relation to Platelet Activation, Glycemic Control and Microvascular Complications in Type 1 Diabetes. Diabetes Care, 36, 1629-1634. https://doi.org/10.2337/dc12-1951

[13] Kodiatte, T.A., Manikyam, U.K., Rao, S.B., et al. (2012) Mean Platelet Volume in Type 2 Diabetes Mellitus. Journal of Laboratory Physicians, 4, 5-9. https://doi.org/10.4103/0974-2727.98662

[14] Robbins, J., Vaccarino, V., Zhang, H. and Kasl, S. (2001) Socioeconomic Status and Type 2 Diabetes in African American and Non-Hispanic White Women and Men: Evidence from the Third National Health and Nutrition Examination Survey. American Journal of Public Health, 91, 76-84. https://doi.org/10.2105/AJPH.91.1.76

[15] National Public Health Survey (1998) Statistics, Canada.

[16] Stelmach, W., Kaczmarczyk-Chalas, K., Bielecki, W. and Drygas, W. (2005) How Education, Income, Control over Life and Lifestyle Contribute to Cardiovascular Risk Factors in Adults in a Post-Communist Country. Public Health, 119, 498-508. https://doi.org/10.1016/j.puhe.2004.09.006

[17] Sarah, H.W., Danijela, G., Caroline, A.J., et al. (2017) Socioeconomic Status and Prevalence of Type 2 Diabetes in Mainland China, Hong Kong and Taiwan: A Systematic Review. Journal of Global Health, 7, Article ID: 011103. https://doi.org/10.7189/jogh.07.011103

[18] Hsu, C.-C., Lee, C.H., Wahlqvist, M.L., Huang, H.-L., Chang, H.-Y., Chen, L., Shih, S.-F., Shin, S.-J., Tsai, W.-C., Chen, T., Huang, C.-T. and Cheng, J.-S. (2012) Poverty Increases Type 2 Diabetes Incidence and Inequality of Care Despite Universal 
Health Coverage. Diabetes Care, 35, 2286-2292. https://doi.org/10.2337/dc11-2052

[19] Carlotta, S., Fulvio, R., Olov, R., Ileana, B., Maria-Dolores, C., Edith, F., Benedetta, B., Eva, A., Larraitz, A., Beverley, B.M.B., Joline, W.J.B., Heiner, B., Françoise, C.C., Francesca, Cr., Blandine, L.-G., Nita, F., Paul, W.F., Valentina, G., Carlos, G., Jytte, H., Anne-Kathrin, I., Rudolf, K., Timothy, K., Kay-Tee, K., Carmen, N., Peter, M.N., Susanne, O.D.T., Kim, O., Valeria, P., Domenico, P., Salvatore, P., Silvia, P., Ramon, Q., Isabelle, R., María-Jose, S., Nadia, S., Ivonne, S., Annemieke, S., Birgit, T., Anne, T., Rosario, T., Daphne, V.D.A., Anne-Claire, V., Patrik, W., Stephen, S., Claudia, L., Elio, R., Paolo, V. and Nicholas, W. (2012) Lower Educational Level Is a Predictor of Incident Type 2 Diabetes in European Countries: The EPIC-Interact Study. International Journal of Epidemiology, 41, 1162-1173.

[20] Flatz, A., Casillas, A., Stringhini, S., Zuercher, E., Burnand, B. and PeytremannBridevaux, I. (2015) Association between Education and Quality of Diabetes Care in Switzerland. International Journal of General Medicine, 8, 87-92. https://doi.org/10.2147/IJGM.S77139

[21] Silvestre, J.S. and Levy, B.I. (2006) Molecular Basis of Angiopathy in Diabetes Mellitus. Circulation Research, 98, 4-6. https://doi.org/10.1161/01.RES.0000200396.90220.41

[22] Shibuya, M. (2001) Structure and Function of VEGF/VEGF-Receptor System Involved in Angiogenesis. Cell Structure and Function, 26, 25-35. https://doi.org/10.1247/csf.26.25

[23] Salven, P., Teerenhovi, L. and Joensuu, H. (1997) A High Pretreatment Serum Vascular Endothelial Growth Factor Concentration Is Associated with Poor Outcome in Non-Hodgkin's Lymphoma. Blood, 90, 3167-3172. https://doi.org/10.1182/blood.V90.8.3167

[24] Brychtova, S., Bezdekova, M., Brychta, T., et al. (2008) The Role of Vascular Endothelial Growth Factors and Their Receptors in Malignant Melanomas. Neoplasma, 55, 273-279.

[25] Shinichiro, O., Takakazu, H., Sadamu, O. and Osamu, T. (2018) The Relationship between Mean Platelet Volume and Fasting Plasma Glucose and HbA1c Levels in a Large Cohort of Unselected Health Check-Up Participants. Journal of Clinical Medicine Research, 10, 345-350. https://doi.org/10.14740/jocmr3361w

[26] Ateş, O., Kiki, I., Bilen, H., Keleş, M., Koçer, I., Kulaçoglu, D.N., et al. (2009) Association of Mean Platelet Volume with the Degree of Retinopathy in Patients with Diabetes Mellitus. European Journal of General Medicine, 6, 99-102. https://doi.org/10.29333/ejgm/82648

[27] Zhou, Z., Ju, H., Sun, M. and Chen, H. (2019) Serum Vascular Endothelial Growth Factor Levels Correlate with Severity of Retinopathy in Diabetic Patients: A Systematic Review and Meta-Analysis. Disease Markers, 2019, Article ID: 9401628. https://doi.org/10.1155/2019/9401628

[28] Wang, J., Xu, X., Elliott, M.H., Zhu, M. and Le, Y.Z. (2010) Muller Cell-Derived VEGF Is Essential for Diabetes-Induced Retinal Inflammation and Vascular Leakage. Diabetes, 59, 2297-2305. https://doi.org/10.2337/db09-1420 